\title{
In vitro and in vivo activity of melflufen (J1) in lymphoma
}

Maryam Delforoush', Sara Strese ${ }^{2}$, Malin Wickström²,3 Rolf Larsson², Gunilla Enblad ${ }^{1}$ and Joachim Gullbo ${ }^{1,2^{*}}$

\begin{abstract}
Background: Melphalan has been used in the treatment of various hematologic malignancies for almost 60 years. Today it is part of standard therapy for multiple myeloma and also as part of myeloablative regimens in association with autologous allogenic stem cell transplantation. Melflufen (melphalan flufenamide ethyl ester, previously called J1) is an optimized derivative of melphalan providing targeted delivery of active metabolites to cells expressing aminopeptidases. The activity of melflufen has compared favorably with that of melphalan in a series of in vitro and in vivo experiments performed preferentially on different solid tumor models and multiple myeloma. Melflufen is currently being evaluated in a clinical phase I/II trial in relapsed or relapsed and refractory multiple myeloma.
\end{abstract}

Methods: Cytotoxicity of melflufen was assayed in lymphoma cell lines and in primary tumor cells with the Fluorometric Microculture Cytotoxicity Assay and cell cycle analyses was performed in two of the cell lines. Melflufen was also investigated in a xenograft model with subcutaneous lymphoma cells inoculated in mice.

Results: Melflufen showed activity with cytotoxic $I_{50}$-values in the submicromolar range (0.011-0.92 $\left.\mu \mathrm{M}\right)$ in the cell lines, corresponding to a mean of 49 -fold superiority $(p<0.001)$ in potency vs. melphalan. In the primary cultures melflufen yielded slightly lower $\mathrm{I}_{50}$-values $(2.7 \mathrm{nM}$ to $0.55 \mu \mathrm{M})$ and an increased ratio vs. melphalan (range 13-455, average 108, $p<0.001$ ). Treated cell lines exhibited a clear accumulation in the G2/M-phase of the cell cycle. Melflufen also showed significant activity and no, or minimal side effects in the xenografted animals.

Conclusion: This study confirms previous reports of a targeting related potency superiority of melflufen compared to that of melphalan. Melflufen was active in cell lines and primary cultures of lymphoma cells, as well as in a xenograft model in mice and appears to be a candidate for further evaluation in the treatment of this group of malignant diseases.

Keywords: J1, Melflufen, Prodrug, Cancer therapeutics, Alkylating agents

\section{Background}

In their pioneering work on nitrogen mustards, Gilman and Philips [1] laid the foundation for modern cancer chemotherapy. These compounds were chemically unstable to hydrolysis and efforts were soon made to increase the stability without reduction of activity. Substitution with electron-withdrawing groups, e.g., a substituted phenyl ring, decreased the rate of aziridinium ion formation and reactivity of the nitrogen mustard [2]. In the early fifties the British scientists Bergel and Stock hypothesized that certain natural amino acids or peptides might, when modified by

\footnotetext{
* Correspondence: Joachim.Gullbo@medsci.uu.se

'Department of Immunology, Genetics and Pathology, Rudbeck Laboratory, Uppsala, Sweden

2Department of Medical Sciences, Section of Clinical Pharmacology, Uppsala University Hospital, Uppsala, Sweden

Full list of author information is available at the end of the article
}

appropriate groups, show anti-tumor activity by interference with the nucleic acid or protein metabolism of malignant cells [3]. In this work the D, L, and DL-form of $p$ bis(2-chloroethyl)aminophenylalanine were synthesized and high activity of the L-form, but only slight activity of the Dform, was demonstrated in a Walker rat carcinoma model $[4,5]$. The lead compound was later named "melphalan", the name being derived from mustard-L-phenylalanine [6]. Intravenous melphalan has single-agent activity in a variety of human malignancies including for example breast cancer, ovarian cancer, testicular cancer and multiple myeloma [7-9]. It was also acknowledged early on, that in poor-prognosis patients with lymphoma, multiple myeloma, or neuroblastoma, high-dose melphalan-containing regimens $\left(>140 \mathrm{mg} / \mathrm{m}^{2}\right)$ yielded both high response rates and improved survival, despite considerable toxicity [10]. 
Melphalan is an important component in the high-dose conditioning chemotherapy regimen most used for lymphoms, BEAM (BCNU, etoposide, cytarabine and melphalan).

Malignant lymphomas are a group of tumors originating from cells of the lymphatic system. The lymphomas are classified according to the WHO-classification into B-cell lymphomas, T-cell lymphomas and Hodgkin lymphoma (HL) and further into many different entities. Of the B-cell lymphomas, diffuse large B-cell lymphoma (DLBCL) is the most common subtype. Systemic chemotherapy is the mainstay of therapy for all lymphomas and approximately $60-70 \%$ of DLBCL patients are cured. In HL even higher cure rates are possible, and the chemotherapy may be combined with radiotherapy. Poor responders and/or early relapse patients at young age and good physical condition are often considered for high dose chemotherapy and stem cell transplantation. Still a significant proportion of lymphoma patients relapse, and there is a need for new drugs to further improve the results and reduce the toxicity of treatment $[11,12]$.

Melflufen (L-melphalanyl-p-L-fluorophenylalanine ethyl ester hydrochloride, previously called J1) is a derivative of the classical alkylating agent melphalan. Chemically melflufen may be described as the ethyl ester of a dipeptide consisting of melphalan and para-fluoro-L-phenylalanine. The drug is susceptible to hydrolysis by aminopeptidases, like aminopeptidase N (APN; also designated CD13) for which melflufen is a substrate [13], that are frequently expressed or overexpressed in tumor tissue [14], providing the molecule with a target directed delivery to cells [15]. It has been demonstrated that melflufen exposure to various malignant cells in vitro results in at least a 10-20 fold higher intracellular concentration of melphalan in comparison with direct treatment with equimolar doses of melphalan $[13,15,16]$. The result is a cytotoxic $\mathrm{IC}_{50}$-value of melflufen significantly lower than for melphalan (10600 -fold lower in various in vitro cell systems) $[15,17,18]$. The advantage of melflufen vs. melphalan has also been demonstrated in various hollow fiber and/or xenograft human tumor models in rodents $[16,19,20]$.

Increased expression of various hydrolytic enzymes like peptidases, esterases and proteases has been described in several types of human malignancies, especially those characterized by fast-growing and aggressive phenotypes [21]. Among these enzymes the metalloproteinase APN has received substantial attention as a marker and mediator of the malignant phenotype, as well as a possible target for anticancerous chemotherapy [14]. It has recently been shown that APN is directly involved in targeted delivery of melflufen resulting in intracellular enrichment of melphalan and subsequent cell death $[16,22]$. APN is commonly expressed in hematopoietic malignancies of myelomonocytic origin and has less commonly been described in lymphoid neoplasms. However, expression of APN has been described in anaplastic large cell lymphoma [23, 24]. In addition, the aminopeptidase inhibitor bestatin (Ubenimex) inhibits the proliferation of histiocytic lymphoma cell line U937 and induces morphological, cytochemical and functional differentiation into monocyte/macrophages [25]. Bestatin has also been evaluated in clinical trials showing therapeutic efficacy and survival benefit in diseases like acute myeloid leukemia (AML) and lymphomas [26].

This study was undertaken to characterize the activity of melflufen against lymphoma cells in vitro and in vivo.

\section{Methods}

\section{Cell lines \& cell culture}

Cytotoxicity was assayed in a panel of twelve human lymphoma cell lines. A brief description of the cell lines is presented in Table 1. The identity of the cell lines was confirmed by department of Laboratory Medicine, Karolinska Institutet in collaboration with department of Oncology and Pathology, Cancer Center Karolinska, Karolinska Institutet, Stockholm, Sweden.

All lymphoma and CCRF-CEM cells were cultured in RPMI 1640 cell growth medium (Sigma-Aldrich, St Louis, MO, USA) supplemented with 10-20\% heat-inactivated fetal calf serum, $2 \mathrm{mM}$ glutamine, $100 \mathrm{U} / \mathrm{mL}$ penicillin, and $100 \mu \mathrm{g} / \mathrm{mL}$ streptomycin (all chemicals from Sigma Aldrich). Cells were grown at $37{ }^{\circ} \mathrm{C}$ in a humidified atmosphere containing $5 \%$ carbon dioxide, split twice weekly and harvested in log-phase for experimental use.

Patient tumor cells from 16 patients with different lymphoma subtypes were also analyzed for in vitro sensitivity (Table 2). The samples were obtained by routine surgery, diagnostic biopsy or bone marrow/peripheral blood sampling. The use of patient samples was approved by the regional Ethics Committee of Uppsala University (Ns 2008/ 246 and 2014/233). Informed consent was waived. Lymphoma cells were isolated from bone marrow or peripheral blood by 1.007 g/ml Ficoll-Paque (Pharmacia Biotech, Uppsala) density gradient centrifugation [27]. Tumor tissue from solid samples was minced into small pieces and tumor cells were then isolated by collagenase dispersion followed by purification on Percoll (Kabi Pharmacia) or Ficoll density gradient centrifugation [28]. Cell viability was determined by trypan blue exclusion test and the proportion of tumor cells in the preparation was judged by inspection of May-Grunwald-Giemsa-stained cytospin preparations by a cytopathologist. Cell culture medium RPMI 1640 (supplemented as described above) was used throughout. In some cases, cells were cryopreserved in a medium containing $10 \%$ dimethylsulfoxide (DMSO, Sigma-Aldrich) and $90 \%$ inactivated calf serum by initial freezing for $24 \mathrm{~h}$ at $-70{ }^{\circ} \mathrm{C}$, followed by storage at $-150{ }^{\circ} \mathrm{C}$. Cryopreservation in this way does not affect drug sensitivity [29]. The characteristics of the primary cultures of human lymphoma cells are listed in Table 1. 
Table 1 List of cell lines used in the study

\begin{tabular}{lllllll}
\hline Cell line & ID & Cell type & Subtype & Resource & Reference & Authentication Y/N \\
\hline DB & ACC539 & DLBCL & GCB & DSMZ, Braunschweig, Germany & {$[34,35]$} & $\mathrm{Y}$ \\
DOHH-2 & ACC47 & DLBCL & GCB & DSMZ, Braunschweig, Germany & {$[36]$} & $\mathrm{N}$ \\
HDLM-2 & ACC 17 & HL & GCB & DSMZ, Braunschweig, Germany & {$[37,38]$} & $\mathrm{N}$ \\
KM-H2 & ACC 8 & HL & GCB & DSMZ, Braunschweig, Germany & {$[37,39]$} & $\mathrm{N}$ \\
L-428 & ACC 197 & HL & GCB & DSMZ, Braunschweig, Germany & {$[37,40]$} & $\mathrm{N}$ \\
OCI-LY3 & ACC 761 & DLBCL & ABC & DSMZ, Braunschweig, Germany & {$[41,42]$} & $\mathrm{Y}$ \\
RC-K8 & ACC561 & DLBCL & ABC & DSMZ, Braunschweig, Germany & {$[41,43]$} & $Y$ \\
SU-DHL-6 & ACC572 & DLBCL & GCB & DSMZ, Braunschweig, Germany & {$[44,45]$} & $Y$ \\
SU-DHL-10 & ACC576 & DLBCL & GCB & DSMZ, Braunschweig, Germany & {$[44,46]$} & $Y$ \\
U-2932 & ACC633 & DLBCL & ABC & DSMZ, Braunschweig, Germany & {$[47,48]$} & $\mathrm{Y}$ \\
U-2940 & ACC634 & DLBCL & PMBL & DSMZ, Braunschweig, Germany & {$[30]$} & Y \\
WSU-NHL & ACC58 & DLBCL & GCB & DSMZ, Braunschweig, Germany & {$[31,34]$} & $\mathrm{N}$ \\
\hline
\end{tabular}

\section{Drugs and chemicals}

Melphalan (Fig. 1a) was obtained as Alkeran ${ }^{\circ}$ from the Swedish Pharmacy (Apoteket AB, Sweden), or bought as a pure chemical from Sigma Aldrich. Melflufen (Fig. 1b, kind gift from Oncopeptides AB, Stockholm, Sweden) was dissolved in DMSO and further diluted in sterile water or phosphate buffered saline (PBS; Sigma-Aldrich). All dilutions with water were made immediately prior to the start of the experiments to minimize the influence of mustard hydrolysis. The standard drugs vincristine, doxorubicin, etoposide, and cytarabine (all from Sigma), as well as 4-

Table 2 Characteristics of primary cells from lymphoma patients

\begin{tabular}{lll}
\hline Sample No. & Diagnosis & Status at analysis \\
\hline 1 & Indolent lymphoma & Refractory \\
2 & Indolent lymphoma & Refractory \\
3 & MCL & Refractory \\
4 & NHL nos & Refractory \\
5 & T-LB & Primary \\
6 & DLBCL & Refractory \\
7 & NHL nos & Refractory \\
8 & FL & Refractory \\
9 & Lymphoma nos & Primary \\
10 & NHL nos & Unknown \\
11 & MCL & Refractory \\
12 & T-PLL & Refractory \\
13 & MCL & Refractory \\
14 & DLBCLtr GC & Refractory \\
15 & DLBCLtr GC & Refractory \\
16 & B-cell lymphoma nos & Primary \\
\hline
\end{tabular}

MCL Mantle cell lymphoma, NHL Non-Hodgkin lymphoma, T-LB Lymphoblastlymphoma of T-cell type, FL Follicular lymphoma, T-PLL Prolymphocytic leukemia of T-cell type, DLBCLtr GC Diffuse large B-cell lymphoma transformed from follicular lymphoma of germinal centre subtype, Nos Not otherwise specified hydroxy-cyclophosphamide (4-HC, the active metabolite of cyclophosphamide, purchased from Niomech - IIT GmbH, Bielefeld, Germany) were dissolved in DMSO and diluted in PBS or sterile water prior to start of the experiments.

\section{The fluorometric microculture cytotoxicity assay FMCA}

Cell viability after drug exposure (cytotoxic efficacy) was analyzed with the fluorometric microculture cytotoxicity assay (FMCA) which has been described in detail previously [30, 31]. The FMCA is a total cell kill assay performed in 96- or 384-well microtiterplates, and based on measurement of fluorescence generated from hydrolysis of fluorescein diacetate (FDA; Sigma-Aldrich). Cell lines were harvested in log-phase and seeded (45 $\mu \mathrm{L} /$ well) into 384-well microtiter plates (Nunc) at a concentration of 100,000 cells $/ \mathrm{ml}$ using pipetting robot Biomek ${ }^{\bullet} 2000$ (Beckman Coulter). The plates were incubated at $37{ }^{\circ} \mathrm{C}$ in humidified atmosphere containing $95 \%$ air and $5 \%$ $\mathrm{CO}_{2}$ for $24 \mathrm{~h}$ followed by the addition of drugs $(2.5 \mathrm{nl}$ portions/well of DMSO drug stock) using Echo 550 Liquid Handler (Labcyte). The plates were further incubated for $72 \mathrm{~h}$. Patient lymphoma cells were handled similarly or seeded ( $45 \mu \mathrm{L} /$ well, 40,000 cells/well) into drug containing ( $5 \mu \mathrm{L} /$ well) 384-well microtiter plates. The plates were incubated at $37{ }^{\circ} \mathrm{C}$, in humidified atmosphere containing $95 \%$ air and $5 \% \mathrm{CO}_{2}$ for $72 \mathrm{~h}$. Each drug concentration was tested in duplicates. Each 384-well microtiter plate had three columns without drugs serving as controls, and one column with medium served as blank. The cells were subsequently washed with PBS and supernatant aspirated leaving $10 \mu \mathrm{l}$ in each well, before the addition of $50 \mu \mathrm{L}, 10 \mu \mathrm{g} / \mathrm{mL}$ FDA. Plates were incubated for $60 \mathrm{~min}$ before measurement of fluorescence $(485 / 538 \mathrm{~nm}$ for excitation and emission respectively) in a Fluostar Omega. The fluorescence generated is proportional to the number of cells in the well with an intact plasma membrane, and data are presented as Survival 
<smiles>CCOC(=O)[C@H](Cc1ccc(F)cc1)NC(=O)[C@H](N)Cc1ccc(N(CCCl)CCCl)cc1</smiles><smiles>NC(Cc1ccc(N(CCCl)CCCl)cc1)C(=O)O</smiles>

Fig. 1 Chemical structure of melflufen (a) and melphalan (b)

Melphalan

Index (SI \%), defined as the mean fluorescence of the test wells expressed as a percentage of control cultures, with blank values subtracted.

Quality criteria for a successful assay included > $75 \%$ starting viability (judged by Trypan blue exclusion), a control signal more than ten times the blank, and finally, a coefficient of variation in control and blank wells of $<30 \%$.

\section{Cell cycle distribution analysis}

Cell cycle distribution analyses were performed on melflufen treated $(0.1,0.2$ and $0.4 \mu \mathrm{M})$ and control cells from the KM-H2 and SU-DHL-10 cell lines in which the cytotoxic activity of melflufen during the $72 \mathrm{~h}$ FMCA assay was calculated as $0.92,0.22$, and $0.71 \mu \mathrm{M}$ respectively (see Table 3 ). To minimize potential artifacts emanating from cells exiting the analyzed cell populations, e.g. due to early and/or extensive cell death, the cells were subjected to basal cell culture conditions for $40 \mathrm{~h}$ (preincubation) followed by 12, 24 and $48 \mathrm{~h}$ treatments with melflufen. Propidium iodide (PI) staining of DNA using Vindelöv's technique was followed by quantitation with flow cytometry analysis. The percentage of cells in each cell cycle phase was determined with the ModFit LT software.

\section{Effect of melflufen in DOHH-2 xenografted mice}

The xenograft study was performed at Pipeline Biotech $\mathrm{A} / \mathrm{S}$, Trige, Denmark and was approved by the national authority "Danish Animal Experiments Inspectorate" (2012-15-2934-00051 C1). The animals had FELASA (Federation for Animal Science Laboratory Associations) SPF-status and the housing and changing systems were designed to assure that the SPF-status was preserved during the study. Diet (Altromin 1324) and water was administered ad libitum.

Table 3 Cytotoxic activity (as $\mathrm{IC}_{50} \mu \mathrm{M}$ ) of standard drugs and melflufen in the panel of cell lines

\begin{tabular}{lllllllcc}
\hline Drugs/Cell lines & Vincristine & Doxorubicin & $4-\mathrm{HC}$ & Etoposide & Cytarabine & Bortezomib & Melphalan & Melflufen \\
\hline DB & 0.013 & 0.052 & 25 & 2.4 & 30 & 0.0020 & 44 & 0.92 \\
DOHH-2 & 0.030 & 0.050 & 22 & 2.3 & 9.6 & 0.0063 & 17 & 0.39 \\
HDLM-2 & 0.0023 & 0.15 & 16 & 3.9 & 0.83 & 0.0031 & 9.3 & 0.088 \\
KM-H2 & 0.0025 & 0.034 & 5.7 & 0.65 & $>100$ & 0.0024 & 8.6 & 0.22 \\
L-428 & 0.14 & 0.089 & 24 & 2.0 & 31 & 0.0044 & 9.4 & 0.73 \\
Ly-3 & 0.0026 & 0.044 & 0.77 & 0.24 & 1.4 & 0.0028 & 0.52 & 0.011 \\
RC-K8 & 0.14 & 0.059 & 21 & 2.7 & 8.7 & 0.0071 & 12 & 0.45 \\
SU-DHL-6 & 0.0014 & 0.053 & 6.7 & 2.7 & 7.0 & 0.0023 & 38 & 0.42 \\
SU-DHL-10 & 0.00026 & 0.19 & 17 & 49 & 0.080 & 0.0047 & 29 & 0.71 \\
U-2932 & 0.0037 & 0.18 & 17 & 3.5 & 0.84 & 0.0038 & 19 & 0.52 \\
U-2940 & 0.020 & 0.026 & 1.6 & 0.34 & 0.91 & 0.0052 & 9.8 & 0.12 \\
WSU-NHL & 0.0015 & 0.032 & 3.8 & 1.5 & 0.17 & 0.0028 & 6.9 \\
\hline
\end{tabular}


Female C.B-17 Scid female mice (nomenclature: C.B-lgh1b Prkdcscid/lcrTac), 5-7 week of age at arrival, were supplied from Taconic-Europe, Denmark, and after 7 days of acclimatization inoculated subcutaneously with $5 \times 10^{6}$ DOHH-2 cells into the right flank. Appearance of tumors was carefully monitored, and the tumors were scored (if not large enough to be measured) or measured three times a week. Tumor diameters were measured in two dimensions using a digital caliper and the volume was estimated by the following formula: $\mathrm{L} \times \mathrm{W} \times 1 / 2 \mathrm{~W}$ (Length $\times$ Width $\times 1 / 2$ Width). Measurements/observations began at day 0 , i.e. the day of inoculation. After randomization on day 21 the animals were allocated to groups and dosed with intravenously injected vehicle $(2 \mathrm{~mL} / \mathrm{kg}$, three times weekly, control group), vincristine $(1 \mathrm{mg} / \mathrm{kg}$, three times weekly, positive control), or melflufen ( $3 \mathrm{mg} / \mathrm{kg}$, two times weekly, test group). Treatment continued for two weeks, and mice were terminated by cervical dislocation at the end of the experiments or at humane endpoints (maximal tumor size, weight loss of $>20 \%$, wounds, etc).

\section{Statistics}

The cytotoxic $\mathrm{IC}_{50}$-vaules (inhibitory concentration $50 \%$ ) for the drugs were determined from log concentrationeffect curves in Graph Pad Prism (GraphPad software Inc., CA, USA) using non-linear regression analysis. Statistical considerations and comparisos between melphalan and melflufen groups were based on $\log \mathrm{IC}_{50}$-values and made with two-sided paired $t$-test, and for three groups one-way ANOVA was used. For comparison between survival curves log-rank test was used. In all tests $p<0.05$ was considered significant.

\section{Results}

\section{Cytotoxic activity in lymphoma cell lines}

The cytotoxic activity of melflufen in human lymphoma cell lines is presented in Table 3 . The calculated $\mathrm{IC}_{50^{-}}$ values varied almost 100 -fold among the cell lines, identifying the DLBCL cell lines Ly-3 as the most sensitive cell line $\left(\mathrm{IC}_{50}\right.$-values for melphalan and melflufen being 0.52 and $0.011 \mu \mathrm{M}$ respectively) and DB as the most resistant ( 44 and $0.92 \mu \mathrm{M}$ ). Melflufen was consistently more potent than melphalan throughout the panel, and the average ratio of $\mathrm{IC}_{50}$-values was 49 -fold (range 12 to 102 , $p<0.001$ ). The pattern of sensitivity among the cell lines was similar for all standard chemotherapeutic drugs. As expected, the correlation coefficients of log IC50-values between the alkylating agents was high (Pearson's correlation $=0.83$ for melflufen vs $4-\mathrm{HC}$, and $=0.88$ for melflufen vs melphalan) in this panel of lymphoma cells. Interestingly, bortezomib deviated from this pattern, and all cell lines appeared sensitive to this drug with comparably small variations in $\mathrm{IC}_{50}$ (range 2.0-7.1 nM).

\section{Cytotoxic activity in primary cultures of human lymphoma cells}

Figure 2 shows the cytotoxicity of melflufen in primary human lymphoma cells, plotted as dose response curves with survival index (SI \%) for each concentration tested. Sensitivity towards melflufen varied considerably $(>300$ fold) and the $\mathrm{IC}_{50}$ range from $2.7 \mathrm{nM}$ to $0.55 \mu \mathrm{M}$. The efficacy of melflufen corresponded to a 13- to 455 -fold potency improvement $(p<0.001)$ compared to melphalan (Table 4).

\section{Effect of treatment with melflufen on cell cycle phase distribution}

The effect of treatment with melflufen on cell cycle phase distribution in KM-H2 and SU-DHL-10 cell lines was analyzed by flow cytometry and illustrates distribution of the cell cycle phases G0/G1, S and G2/M. After a $48 \mathrm{~h}$ treatment with melflufen, a clear accumulation of cells in the G2/M phase was seen, and this response was detected in both cell lines tested (Fig. 3). However it was much more pronounced in SU-DHL-10 cell line. In SUDHL-10 cells, G2 arrest was already seen after $24 \mathrm{~h}$ of treatment with melflufen. For KM-H2 cells, 48 h were needed. In all tested concentrations a dramatic increase in G2 after $48 \mathrm{~h}$ of treatment was seen which is consistent with the cells trying to divide yet unable to do it due to DNA damage and thus arresting in G2.

\section{Activity in DOHH-2 xenografts}

Melflufen significantly inhibited growth of the subcutaneously xenografted DOHH-2 lymphoma tumors during the treatment period (Fig. 4a) and prolonged survival (Fig. 4b) compared to the vehicle treated control. Premature sacrifice in the vehicle treated control group but

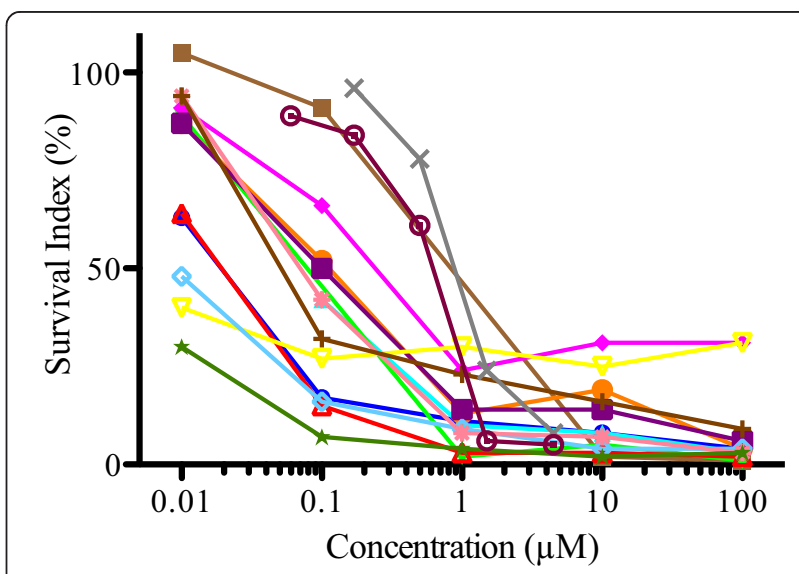

Fig. 2 Activity of melflufen in primary lymphoma cells. The cytotoxicity of melflufen in human primary lymphoma cells, after incubation for 72 h, was tested by the Fluorometric Cytotoxicity Assay. Each dose response curve is one patient cell culture, plotted as survival index (\%) as a function of concentration 
Table $4 \mathrm{IC}_{50}(\mu \mathrm{M})$ for melphalan and melflufen, and melphalan/ melflufen ratio in primary human lymphoma cells

\begin{tabular}{llll}
\hline Patient no & $I_{50}$ melphalan $(\mu \mathrm{M})$ & $I_{50}$ melflufen $(\mu \mathrm{M})$ & Ratio \\
\hline 1 & 1.7 & 0.018 & 94 \\
2 & 6.7 & 0.51 & 13 \\
3 & 7.0 & 0.059 & 118 \\
4 & 19 & 0.053 & 363 \\
5 & 89 & 0.55 & 162 \\
6 & 8.5 & 0.12 & 73 \\
7 & 29 & 0.11 & 271 \\
8 & 6.0 & 0.018 & 337 \\
9 & 2.8 & $<0.01$ & 278 \\
10 & 0.73 & 0.0080 & 91 \\
11 & 13 & 0.079 & 160 \\
12 & 1.2 & 0.0027 & 455 \\
13 & 31 & 0.080 & 388 \\
14 & $\mathrm{NA}$ & $\mathrm{NA}$ & $\mathrm{NA}$ \\
15 & 24 & 0.90 & 27 \\
16 & 16 & 0.66 & 24 \\
MEAN & 8.4 & 0.078 & 108 \\
\hline Th & 24 & &
\end{tabular}

The potency difference between melphalan and melflufen was statistically significant $(p<0.001$, paired 2-tailed $t$-test) not in the melflufen group was done due to large tumors (4 of 5 animals). All remaining animals were terminated on day 37. Treatment with melflufen intravenously on a twice-weekly schedule at the dose $3 \mathrm{mg} / \mathrm{kg}$ had no detectable effect on animal health or weight development during the experiment, and all animals survived until termination. In contrast, the positive control group (treated with vincristine) presented significant body weight loss, and two animals reached the toxicity endpoint $(-20 \%$ body weight) at or before the end of the experiment (Fig. 4c).

\section{Discussion}

Melphalan has been used for almost sixty years in the treatment of a broad spectrum of malignancies and is part of many combinations regimes. Melphalan belongs to a class of agents, i.e. alkylators, which exert cytotoxic action through covalent interaction with intracellular nucleophiles, especially DNA. Difunctional agents, able to crosslink a DNA strand within a double helix (intrastrand), between two strands (interstrand) or between DNA and proteins, are usually more active than monofunctional agents. Although covalent adduct formation is the mechanism of cytotoxicity common to all the antitumor alkylating agents, these drugs have widely different potency, toxicity and disease selectivity. Differences in the non-alkylating
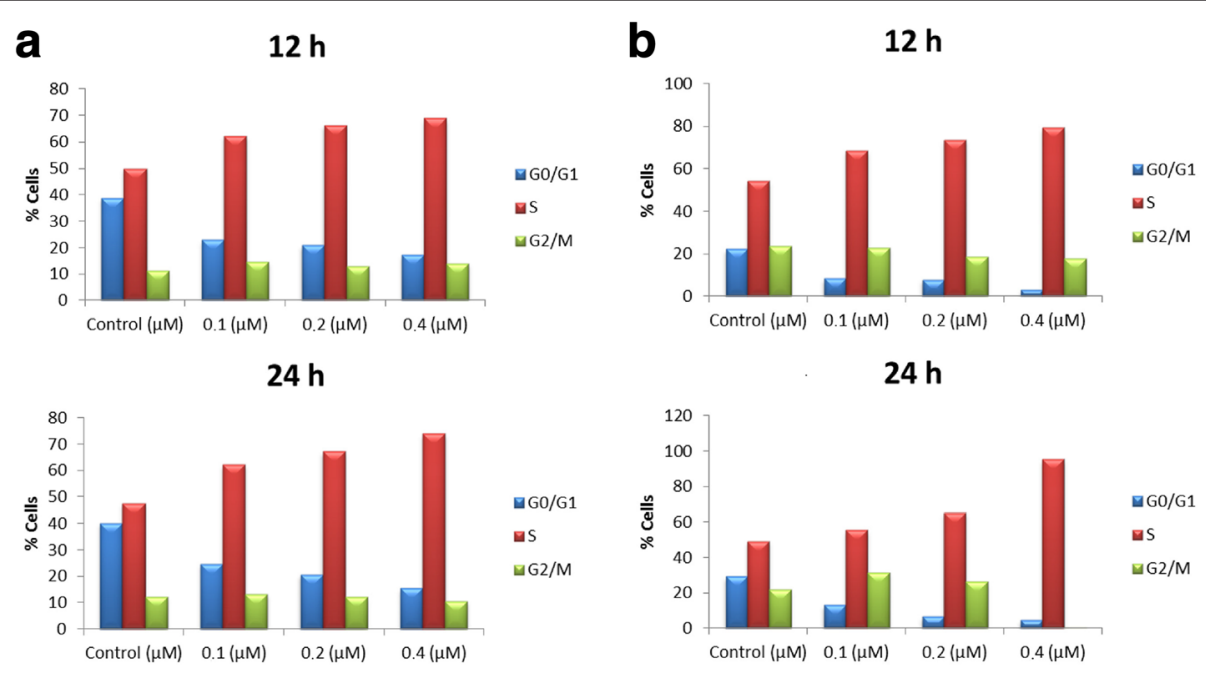

$48 \mathrm{~h}$

$48 \mathrm{~h}$
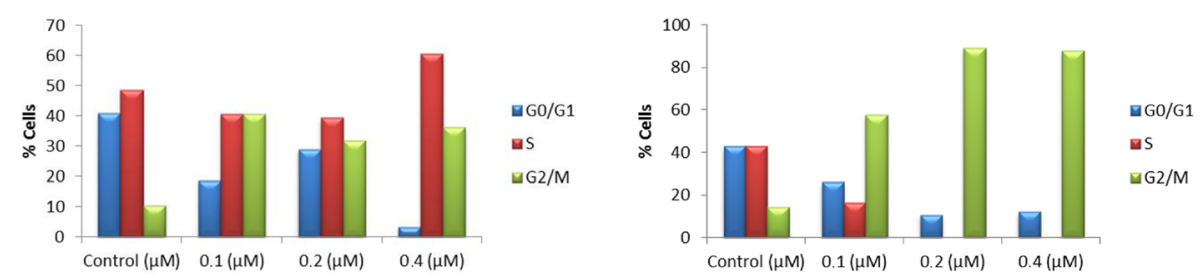

Fig. 3 Effects of melflufen on cell cycle phase distribution. KM-H2 (a) and SU-DHL-10 (b) cell lines were incubated for $40 \mathrm{~h}$ during basal conditions before treatment with melflufen for 12, 24 and $48 \mathrm{~h}$. The analyses were performed by flow cytometry and show distribution of the cell cycle phases $\mathrm{G} 0 / \mathrm{G} 1, \mathrm{~S}$ and $\mathrm{G} 2 / \mathrm{M}$ resulting from treatment with melflufen 0.1-0.4 $\mu \mathrm{M}$ 

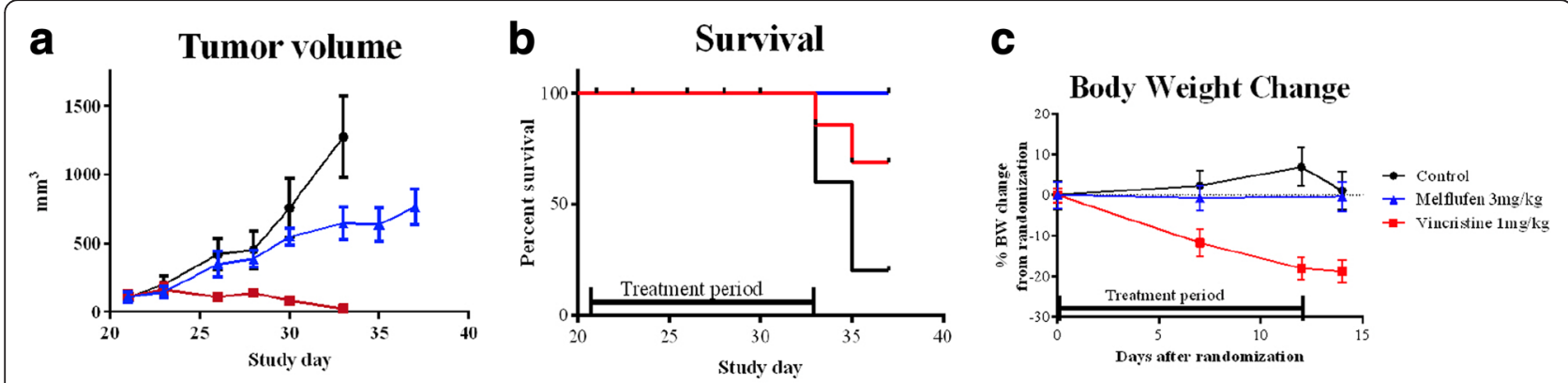

Fig. 4 Effects of melflufen in subcutaneous DOHH-2 xenografted mice. Presented as tumor volume (a), survival (b) and body weight change (c). Mice in control group $(n=5)$ gained weight and showed no signs of toxicity, but individuals were prematurely sacrificed due to tumor size on day $33(n=2)$ and $35(n=2)$. Animals in vincristine control group had excellent tumor control but lost weight and one animal was sacrificed on day 25, one on day 33 due to $>20 \%$ weight reduction. Animals treated with melflufen had no weight loss but significant tumor growth reduction on day 33 ( $p<0.05)$. Log-rank test showed a significant difference in survival between the control group and the melflufen treated group ( $p=0.0144)$. In A and $C$ mean values with SEM are displayed

portions of these molecules probably lead to different biodistribution and normal tissue toxicity of these drugs [8]. New representatives of this class of drugs are continuously introduced, such as temozolomide and bendamustin.

The clinical use of most chemotherapeutic agents is limited by the associated toxicity, often originating from organs with high proliferation (e.g. bone marrow). Many attempts have been made to, more or less, specifically target the tumor cells or the tumor microenvironment, for example, the use of a prodrug approach where the prodrug is selectively activated in tumors overexpressing the prodrug-activating enzyme(s) [32]. Many such targets have been suggested in the literature, among them different aminopeptidases like APN, which is described as overexpressed in many malignant tissues making it a possible and attractive target for cancer chemotherapy [14]. Melflufen is a dipeptide derivative carrying melphalan as one of its amino acid moieties. Compelling evidence shows that the activity benefit of melflufen compared to melphalan originates from APN dependent intracellular cleavage of melflufen yielding high concentrations of melphalan in the cytoplasm, i.e. a targeted delivery $[14,16,22]$.

$\mathrm{APN} / \mathrm{CD} 13$ is commonly expressed in hematopoietic malignancies of myelomonocytic origin and has less commonly been described in lymphoid neoplasms. However, effects of the aminopeptidase inhibitor bestatin on lymphoma cells have been shown in vitro [26], and the effects of this drug in lymphoma patients have also been evaluated in clinical trials [26, 33]. Wickstrom et al. [18] investigated the effects of melflufen in primary cultures of tumor cells from 176 patients with various diagnoses. Among these were fourteen patients with lymphoma, and the in vitro activity difference between melphalan and melflufen was in this diagnosis exceptionally high $(160 \times)$ prompting further investigation of the activity of melflufen in lymphoma.

The results presented here show that melflufen is indeed an active drug both in Hodgkin and non-Hodgkin lymphoma cell lines in vitro, yielding $\mathrm{IC}_{50}$-values in the range of $11 \mathrm{nM}$ to $0.92 \mu \mathrm{M}$. A similar pattern was found in patient cells $\left(\mathrm{IC}_{50}\right.$ of $2.7 \mathrm{nM}$ to $\left.0.55 \mu \mathrm{M}\right)$ clearly suggesting that lymphoma is a possible clinical diagnosis for future use of melflufen. The in vivo xenograft model study indicated modest activity of melflufen in the dose and regimen used ( $3 \mathrm{mg} / \mathrm{kg}$ on a twice weekly schedule), but on the other hand, without any signs of toxicity the dose and/or intensity of the treatment schedule could most probably be increased. Animals in the positive control group suffered severely from toxicity, and albeit excellent tumor control, some of them had to be prematurely sacrificed due to treatment associated weight loss.

\section{Conclusion}

The conclusion of this study demonstrates that melflufen is an active drug against lymphoma in vitro and in vivo and further evaluation in this diagnosis seems warranted.

\section{Ethics approval and consent to participate}

The xenograft study was performed at Pipeline Biotech A/S, Trige, Denmark and was approved by the national authority "Danish Animal Experiments Inspectorate" (2012-15-2934-00051 C1).

The use of patient samples was approved by the regional Ethics Committee of Uppsala University (Ns 2008/246 and 2014/233).

The use of the patients' samples in research was approved without written informed consent of the patients. The reasons why the ethical committee waived informed consent were that the patients had approved storing of samples in the Uppsala biobank and that these samples could be used for research after ethical approval without contacting the patient if the committee found the research of good quality and not compromising integrity of the patient. Furthermore, all patients included in this 
study have given an oral informed consent but that was not a requirement of the ethical committee.

\section{Availability of data and materials}

\section{The datasets supporting the conclusions of this article} are included within the article.

\section{Abbreviations}

HL: Hodgkin lymphoma; DLBCL: Diffuse large B-cell lymphoma; AML: Acute myeloid leukemia; DMSO: Dimethylsulfoxide; PBS: Phosphate buffered saline; 4-HC: 4-hydroxy-cyclophosphamide; FMCA: Fluorometric microculture cytotoxicity assay; FDA: Fluorescein diacetate; SI: Survival index; PI: Propidium iodide; MCL: Mantle cell lymphoma; NHL: NonHodgkin lymphoma; T-LB: Lymphoblastlymphoma of T-cell type FL: Follicular lymphoma; T-PLL: Prolymphocytic leukemia of T-cell type; DLBCLtr GC: Diffuse large B-cell lymphoma transformed from follicular lymphoma of germinal centre subtype; Nos: Not otherwise specified.

\section{Competing interests}

$R L$ and JG are co-founders and minor shareholders of Oncopeptides $A B$, a small Swedish research and development company currently evaluating the effects of melflufen in clinical trials.

\section{Authors' contributions}

MD handled cell lines and performed FMCA and cell cycle analysis experiments. SS and MW summarized results from primary cultures and assisted in FMCA experiments. GE is head of the Oncology lab and participated in planning of the study. RL is head of Clinical Pharmacology lab and participated in planning of the study. JG designed the study including the xenograft experiment performed at a $\mathrm{CRO}$, collected results and wrote the first manuscript. All authors have commented on the draft and participated in the finalization of the manuscript. All authors read and approved the final manuscript.

\section{Acknowledgement}

The skillful technical assistance from Lena Lenhammar, Christina Leek, Carina Alvfors, Kristin Blom, Annika Jonasson and Anna-Karin Lannergård is gratefully acknowledged.

\section{Author details}

'Department of Immunology, Genetics and Pathology, Rudbeck Laboratory, Uppsala, Sweden. ${ }^{2}$ Department of Medical Sciences, Section of Clinical Pharmacology, Uppsala University Hospital, Uppsala, Sweden. ${ }^{3}$ Department of Women's and Children's Health, Karolinska Institutet, Childhood Cancer Research Unit, Stockholm, Sweden.

\section{Received: 9 June 2015 Accepted: 31 March 2016}

\section{Published online: 04 April 2016}

\section{References}

1. Gilman A, Philips FS. The biological actions and therapeutic applications of the B-chloroethyl amines and sulfides. Science. 1946;103(2675):409-15.

2. Haddow A, Kon GAR, Ross WCJ. Effects upon tumours of various haloalkylarylamines. Nature. 1948:162:824-5.

3. Bergel F, Stock JA. Cyto-active Amino-acid and Peptide Derivatives. Part I. Substituted Phenylalanines. J Chem Soc. 1954: 2409-17

4. Bergel F, Stock JA. Cytotoxic alpha amino acids and peptides. Br Emp Cancer Camp Annu Rep. 1951:31:6-7.

5. Bergel F, Burnop VCE Stock JA. Cyto-active Amino-acids and Peptides. Part II. Resolution of Substituted Phenylalanines and Synthesis of p-Di(2-chloroethyl)amino-DL-phenyl[B-14C]alanine. J Chem Soc. 1955:1223-9

6. Bergel F, Stock JA. Cyto-active Amino-acids and Peptides. Part VIII. N(alpha)-Acyl, Amide, Ester and Peptide Derivatives of Melphalan. J Chem Soc. 1960:3658-69

7. Furner RL, Brown RK. L-phenylalanine mustard (L-PAM): the first 25 years. Cancer Treat Rep. 1980:64(4-5):559-74

8. Teicher BA. Antitumor alkylating agents. In: DeVita VT, Hellman S, Rosenberg SA editors. Cancer: Principles \& Practise of Oncology (on CD-ROM). Philadelphia, Pennsylvania: Lippincott-Raven Publishers; 1997.

9. Jones RB. Clinical pharmacology of melphalan and its implications for clinical resistance to anticancer agents. Cancer Treat Res. 2002;112:305-22.
10. Sarosy $\mathrm{G}$ et al. The systemic administration of intravenous melphalan. J Clin Oncol. 1988:6(11):1768-82

11. Bakshi N, Maghfoor I. The current lymphoma classification: new concepts and practical applications triumphs and woes. Ann Saudi Med. 2012;32(3):296-305.

12. Siegel $R$ et al. Cancer statistics, 2014. CA Cancer J Clin. 2014;64(1):9-29.

13. Wickström $M$ et al. The alkylating prodrug J1 can be activated by aminopeptidase $\mathrm{N}$, leading to a possible target directed release of melphalan. Biochem Pharmacol. 2010;79:1281-90.

14. Wickstrom M, Larsson R, Nygren P, et al. Aminopeptidase N (CD13) as a target for cancer chemotherapy. Cancer Sci. 2011;102:501-8.

15. Gullbo J et al. Activity of hydrolytic enzymes in tumour cells is a determinant for anti-tumour efficacy of the melphalan containing prodrug J1. J Drug Target. 2003;11(6):355-63.

16. Chauhan D, Ray A, Viktorsson K, et al. In Vitro and In Vivo Antitumor Activity of a Novel Alkylating Agent, Melphalan-Flufenamide, against Multiple Myeloma Cells. Clin Cancer Res. 2013;19:3019-31.

17. Gullbo J et al. Antitumor activity of the alkylating oligopeptides J1 (L-melphalanyl-p-L-fluorophenylalanine ethyl ester) and P2 (L-prolylm-L-sarcolysyl-p-L-fluorophenylalanine ethyl ester): comparison with melphalan. Anticancer Drugs. 2003;14(8):617-24.

18. Wickstrom $\mathrm{M}$ et al. The novel alkylating prodrug J1: diagnosis directed activity profile ex vivo and combination analyses in vitro. Invest New Drugs. 2008;26(3):195-204

19. Gullbo J et al. Antitumor efficacy and acute toxicity of the novel dipeptide melphalanyl- p -L-fluorophenylalanine ethyl ester (J1) in vivo. Invest New Drugs. 2004;22(4):411-20.

20. Wickstrom $\mathrm{M}$ et al. The novel melphalan prodrug J1 inhibits neuroblastoma growth in vitro and in vivo. Mol Cancer Ther. 2007:6(9):2409-17.

21. Dubowchik GM, Walker MA. Receptor-mediated and enzyme-dependent targeting of cytotoxic anticancer drugs. Pharmacol Ther. 1999:83(2):67-123.

22. Wickstrom $\mathrm{M}$ et al. The alkylating prodrug $\mathrm{J} 1$ can be activated by aminopeptidase $\mathrm{N}$, leading to a possible target directed release of melphalan. Biochem Pharmacol. 2010;79(9):1281-90

23. Kesler MV et al. Anaplastic large cell lymphoma: a flow cytometric analysis of 29 cases. Am J Clin Pathol. 2007;128(2):314-22.

24. Dunphy $\mathrm{CH}$ et al. CD30+ anaplastic large-cell lymphoma with aberrant expression of CD13: case report and review of the literature. J Clin Lab Anal. 2000;14(6):299-304.

25. Murata $\mathrm{M}$ et al. Effect of ubenimex on the proliferation and differentiation of U937 human histiocytic lymphoma cells. Leukemia. 1994;8(12):2188-93.

26. Bauvois B, Dauzonne D. Aminopeptidase-N/CD13 (EC 3.4.11.2) inhibitors: chemistry, biological evaluations, and therapeutic prospects. Med Res Rev. 2006:26(1):88-130.

27. Larsson $\mathrm{R}$ et al. Laboratory determination of chemotherapeutic drug resistance in tumor cells from patients with leukemia, using a fluorometric microculture cytotoxicity assay (FMCA). Int J Cancer. 1992;50(2):177-85.

28. Csoka K et al. Cytotoxic drug sensitivity testing of tumor cells from patients with ovarian carcinoma using the fluorometric microculture cytotoxicity assay (FMCA). Gynecol Oncol. 1994;54(2):163-70.

29. Nygren $P$ et al. Feasibility of the fluorometric microculture cytotoxicity assay (FMCA) for cytotoxic drug sensitivity testing of tumor cells from patients with acute lymphoblastic leukemia. Leukemia. 1992;6(11):1121-8.

30. Lenz $\mathrm{G}$ et al. Aberrant immunoglobulin class switch recombination and switch translocations in activated B cell-like diffuse large B cell lymphoma. J Exp Med. 2007;204(3):633-43.

31. Sambade C et al. U-2940, a human B-cell line derived from a diffuse large cell lymphoma sequential to Hodgkin lymphoma. Int J Cancer. 2006;118(3): 555-63.

32. Huang PS, Oliff A. Drug-targeting strategies in cancer therapy. Curr Opin Genet Dev. 2001:11(1):104-10.

33. Arimori $\mathrm{S}$ et al. The effect of bestatin on patients with acute and chronic leukemia and malignant lymphoma. Tokai J Exp Clin Med. 1980;5(1):63-71.

34. Ngo VN et al. Oncogenically active MYD88 mutations in human lymphoma. Nature. 2011:470(7332):115-9.

35. Morin RD et al. Somatic mutations altering EZH2 (Tyr641) in follicular and diffuse large B-cell lymphomas of germinal-center origin. Nat Genet. 2010; 42(2):181-5

36. Kluin-Nelemans $\mathrm{HC}$ et al. A new non-Hodgkin's B-cell line $(\mathrm{DoHH} 2)$ with a chromosomal translocation t(14;18)(q32;q21). Leukemia. 1991;5(3):221-4.

37. Drexler HG. Recent results on the biology of Hodgkin and Reed-Sternberg cells. II. Continuous cell lines. Leuk Lymphoma. 1993;9(1-2):1-25. 
38. MacLeod RA et al. Karyotypic dissection of Hodgkin's disease cell lines reveals ectopic subtelomeres and ribosomal DNA at sites of multiple jumping translocations and genomic amplification. Leukemia. 2000;14(10):1803-14.

39. Kamesaki $\mathrm{H}$ et al. Cytochemical, immunologic, chromosomal, and molecular genetic analysis of a novel cell line derived from Hodgkin's disease. Blood. 1986;68(1):285-92.

40. Schaadt $\mathrm{M}$ et al. Two neoplastic cell lines with unique features derived from Hodgkin's disease. Int J Cancer. 1980;26(6):723-31.

41. Kubonishi I et al. Characterization of a new human lymphoma cell line (RC-K8) with t(11;14) chromosome abnormality. Cancer. 1986;58(7):1453-60.

42. Tweeddale ME et al. The presence of clonogenic cells in high-grade malignant lymphoma: a prognostic factor. Blood. 1987;69(5):1307-14.

43. Kubonishi I, Niiya K, Miyoshi I. Establishment of a new human lymphoma line that secretes plasminogen activator. Jpn J Cancer Res. 1985;76(1):12-5.

44. Yee $\mathrm{C}$ et al. A possible autocrine role for interleukin-6 in two lymphoma cell lines. Blood. 1989;74(2):798-804.

45. Epstein AL et al. Biology of the human malignant lymphomas. IV. Functional characterization of ten diffuse histiocytic lymphoma cell lines. Cancer. 1978; 42(5):2379-91.

46. Hecht BK et al. Histiocytic lymphoma cell lines: immunologic and cytogenetic studies. Cancer Genet Cytogenet. 1985;14(3-4):205-18.

47. Epstein AL, Kaplan HS. Feeder layer and nutritional requirements for the establishment and cloning of human malignant lymphoma cell lines. Cancer Res. 1979;39(5):1748-59.

48. Amini RM et al. A novel B-cell line (U-2932) established from a patient with diffuse large B-cell lymphoma following Hodgkin lymphoma. Leuk Lymphoma. 2002;43(11):2179-89.

\section{Submit your next manuscript to BioMed Central and we will help you at every step:}

- We accept pre-submission inquiries

- Our selector tool helps you to find the most relevant journal

- We provide round the clock customer support

- Convenient online submission

- Thorough peer review

- Inclusion in PubMed and all major indexing services

- Maximum visibility for your research

Submit your manuscript at www.biomedcentral.com/submit

C Biomed Central 